INR-TH-2016-042

\title{
Hadronically decaying heavy dark matter and high-energy neutrino limits
}

\author{
M. Yu. Kuznetsov* \\ Institute for Nuclear Research of the Russian Academy of Sciences, \\ 60th October Anniversary Prospect 7a, 117312 Moscow, Russia
}

\begin{abstract}
We consider dark matter consisting of long-living particles with masses $10^{7} \lesssim M \lesssim 10^{16} \mathrm{GeV}$ decaying through hadronic channel as a source of high energy neutrino. Using recent data on high energy neutrino from IceCube and Pierre Auger experiments we derive the upper-limits on neutrino flux from dark matter decay and constraints on dark matter parameter space. For the dark matter masses of order $10^{8} \mathrm{GeV}$ the constraints derived are slightly stronger than those obtained for the same dark matter model using the high energy gamma-ray limits.
\end{abstract}

Keywords: heavy dark matter, neutrino.

\section{Introduction}

The idea that dark matter consists of heavy long-living particles was proposed in the context of inflationary cosmology. There are several mechanisms of production of these particles that are able to yield the observed relic abundance. Among them are production in non-equilibrium plasma, production during the decay of inflaton (preheating) and production by non-stationary gravitational field [1-11]. Although, heavy dark matter was also discussed irrespectively of inflation $[12-14]$. It was also realised that heavy decaying particles can be the source of ultra high energy cosmic rays (UHECRs) that evade the GZK cutoff [3, 4]. Although, the absence of the GZK cutoff is not confirmed by the modern cosmic-ray experiments [15, 16 the heavy dark matter is still under consideration as a possible source of high energy cosmic rays, in particular photons and neutrino.

The heavy dark matter candidate $X$ has two main parameters: mass $M_{X}$ and lifetime $\tau$. The case of absolutely stable $X$-particles is not so interesting from the experimental point of view - its annihilation cross-section is bounded by unitarity: $\sigma_{X}^{\text {ann. }} \sim 1 / M_{X}^{2}$, making their indirect detection impossible for the today experiments [17]. The direct detection of these particles, whether stable or long-living, is also experimentally unreachable due to their small number density. However, there are several sources of

\footnotetext{
*mkuzn@inr.ac.ru
} 
constraints for the heavy dark-matter parameters: the mass is subject to cosmological constraints $8,9,18,21$, and the lifetime of the dark-matter particles can be effectively constrained using the observed fluxes of various high energy particles or limit on these fluxes. For example, in Ref. 22] the constraints have been put using the shape of charged cosmic-ray spectra. However, with the modern cosmic ray data this method bounds $\tau$ not so well as gamma-ray and neutrino flux limits. Various gamma-ray data and limits was employed to constrain heavy dark-matter parameters in Refs. [23 26, 41].

The detection of the high energy neutrino events by IceCube experiment [27, 28] has attracted significant attention. There were many works interpreting these events as an astrophysical neutrino signal [29 31] as well as a dark matter decay signal [24,32,36]. At the same time, the constraints on various models of neutrino origin have been placed [37]. There were also pre-IceCube studies where neutrino limits were employed to constrain heavy dark matter parameters $[23,38]$. This study is mainly inspired by the publication of the new refined sample of the IceCube high-energy neutrino data along with the updated exposure of this experiment [39]. In that work the stringent cuts were employed to eliminate the atmospheric neutrino background. The resulting data set contains only two events with PeV order energy, both consistent with the astrophysical neutrino MonteCarlo. This fact together with the non-observation of higher energy events allows the IceCube collaboration to place limits on the astrophysical neutrino flux and to constrain several models of astrophysical neutrino origin.

In this work we use the same data sample to place limits on the neutrino flux from the decay of dark matter with masses $10^{7} \lesssim M_{X} \lesssim 10^{16} \mathrm{GeV}$ and to constrain its lifetime. For comparison we also derive constrains using Pierre Auger Observatory data [40] that reports non-detection of neutrino with energies $E_{\nu} \gtrsim 10^{17} \mathrm{eV}$. This study complements our previous research [41], where heavy decaying dark matter parameters was constrained by the high energy gamma-ray limits.

\section{Neutrino flux from dark matter decay}

In this study we consider dark matter consisting of heavy scalars $X$ decaying through the channel $X \rightarrow q \bar{q} \rightarrow \nu_{i}\left(\bar{\nu}_{i}\right)$. We assume that all quark flavors are coupled to $X$ similarly. The decay through this channel can be described irrespectively of the particular form of $X$-quarks coupling, since the most important physical phenomenon of relevance is hadronisation, see Refs. [43, 44 for the details of this approach. It should be noted that other possible decay modes, e.g. those related to gauge bosons, may also lead to comparable neutrino flux, however we do not consider theses modes in the present study. Some results and constraints related to heavy dark matter decaying into neutrino via various channels can be found in Refs. [23, 26, 38, 42]. The main difference between the present study and these works is that we consider the DGLAP evolution of the fragmentation functions (see below) that allows us to handle the wider range of the dark matter masses.

The method of calculation of the final state stable particles spectra for the hadronic decays of heavy particles was reviewed in our previous work [41] and mainly follows the Refs. [43, 44]. In this study we consider the neutrino flux. The main contribution to the 
flux comes from the decay of charged pions via processes

$$
\pi \rightarrow \mu \nu_{\mu}, \quad \mu \rightarrow e \nu_{\mu} \nu_{e}
$$

There are also contributions from kaons as well as from charmed mesons but they are an order of magnitude smaller than the contribution of pions. Moreover, the uncertainty of the pion flux which is dominated by the uncertainty of the pion fragmentation functions on the initial energy scale is of the same order as the contributions of other mesons to the neutrino flux [45]. Therefore we assume that the neutrino production is saturated by the pion decays. Using the results of Ref. [42] we are also make sure of negligibility of electro-weak corrections to the decay spectrum.

We consider the spectrum of pions $\frac{d N_{\pi}}{d x}$, where $x=\frac{2 E_{\pi}}{M_{X}}$, produced in the decay of $M_{X}$. It can be obtained by the evolution of the pion fragmentation functions from the initial scale to the $M_{X}$ scale via DGLAP equations [46, 47]:

$$
\frac{\partial D_{i}^{\pi}(x, s)}{\partial \ln s}=\sum_{j} \frac{\alpha_{s}(s)}{2 \pi} P_{i j}\left(x, \alpha_{s}(s)\right) \otimes D_{j}^{\pi}(x, s),
$$

where $D_{i}^{\pi}(x, s)$ is the fragmentation function of pion from the parton $i, s$ is the factorization scale, $\otimes$ denotes the convolution $f(x) \otimes g(x) \equiv \int_{x}^{1} d z / z f(z) g(x / z)=\int_{x}^{1} d z / z f(x / z) g(z)$ and $P_{i j}(x, s)$ is the splitting function for the parton branching $i \rightarrow j$. We use the same assumptions about DGLAP evolution and fragmentation functions as in our previous work [41], namely we assume that all quark flavors are coupled to gluon similarly and consider the mixing of gluon fragmentation function with the quark singlet fragmentation function. As in our previous work we use the code of Ref. [43] to solve DGLAP equations numerically in the leading order of $\alpha(s)$. We take the initial fragmentation functions parametrized on the scale of $1 \mathrm{GeV}$ from Ref. [45] and extrapolate them to the interval $10^{-5} \leq x \leq 1$.

The neutrino spectrum from pions decay is given by

$$
\frac{d N_{\pi \rightarrow \nu_{\mu}}}{d x}=2 R \int_{x R}^{1} \frac{d y}{y} \frac{d N_{\pi}}{d y}
$$

while the neutrino spectrum from the decay of secondary muons is

$$
\frac{d N_{\mu \rightarrow \nu_{i}}}{d x}=2 \int_{x}^{1} \frac{d z}{z} f_{\nu_{i}}\left(\frac{y}{z}\right) \frac{d N_{\pi}}{d z}
$$

where $r=\left(m_{\mu} / m_{\pi}\right)^{2} \simeq 0.573, R=\frac{1}{1-r}$ and the functions $f_{\nu_{i}}(x)$ are taken from Ref. [48]:

$$
\begin{gathered}
f_{\nu_{i}}(x)=g_{\nu_{i}}(x) \Theta(x-r)+\left(h_{\nu_{i}}^{(1)}(x)+h_{\nu_{i}}^{(2)}(x)\right) \Theta(r-x), \\
g_{\nu_{\mu}}(x)=\frac{3-2 r}{9(1-r)^{2}}\left(9 x^{2}-6 \ln x-4 x^{3}-5\right), \\
h_{\nu_{\mu}}^{(1)}(x)=\frac{3-2 r}{9(1-r)^{2}}\left(9 r^{2}-6 \ln r-4 r^{3}-5\right)
\end{gathered}
$$




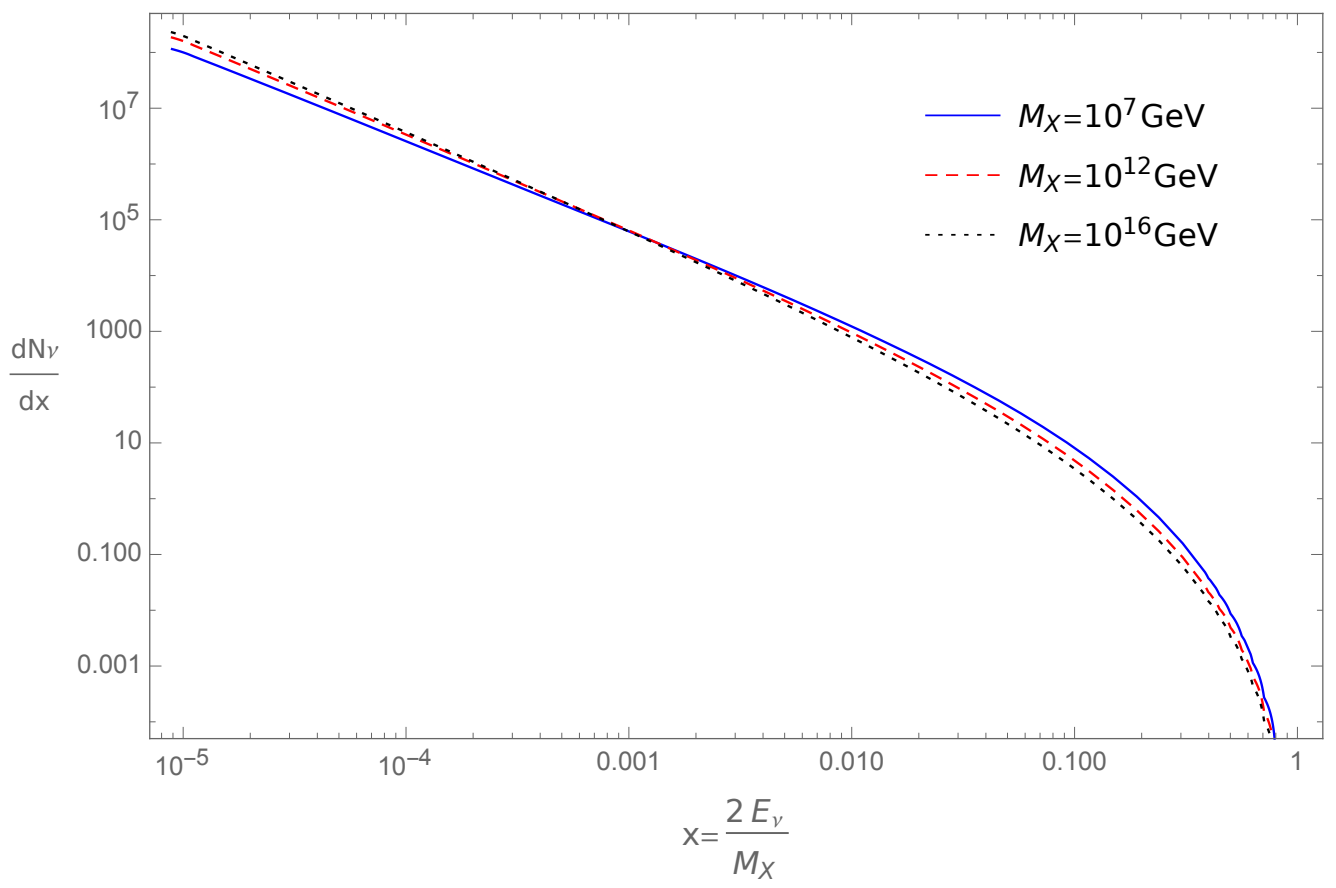

Figure 1: The total spectra of neutrino and antineutrino from $X$ particle decay for three different values of $M_{X}$.

$$
\begin{gathered}
h_{\nu_{\mu}}^{(2)}(x)=\frac{(1+2 r)(r-x)}{9 r^{2}}\left[9(r+x)-4\left(r^{2}+r x+x^{2}\right)\right], \\
g_{\nu_{e}}(x)=\frac{2}{3(1-r)^{2}}\left[(1-x)\left(6(1-x)^{2}+r\left(5+5 x-4 x^{2}\right)\right)+6 r \ln x\right], \\
h_{\nu_{e}}^{(1)}(x)=\frac{2}{3(1-r)^{2}}\left[(1-r)\left(6-7 r+11 r^{2}-4 r^{3}\right)+6 r \ln r\right], \\
h_{\nu_{e}}^{(2)}(x)=\frac{2(r-x)}{3 r^{2}}\left(7 r^{2}-4 r^{3}+7 x r-4 x r^{2}-2 x^{2}-4 x^{2} r\right) .
\end{gathered}
$$

The examples of neutrino spectra from the decay of $X$ particles with different masses are shown in Fig. 1.

Neutrinos propagate cosmological distances unattenuated. The resulting flux that reaches the Earth consists of the galactic and extragalactic parts. The initial flavor composition of the pion decay products is modified by the neutrino oscillations during the propagation. We assume the flux reaching the Earth is completely mixed, i.e. the flavor ratio $\nu_{e}: \nu_{\mu}: \nu_{\tau}$ is $1: 1: 1$. We also assume that neutrinos are radiated isotropically in the decay of $X$ particle. For the galactic neutrino flux calculation we use the NavarroFrenk-White dark matter distribution [49,50] with the parametrization for the Milky Way from Ref. [42]. Being strongly anisotropic, the galactic signal has to be convolved with the exposure of the particular experiment to obtain the perceived flux (see next Section). Contrary, the extragalactic flux is isotropic and undergoes the cosmological redshifting

$$
\frac{d N_{\nu}^{\mathrm{EG}}}{d E_{\nu}}\left(E_{\nu}\right)=\frac{1}{4 \pi M_{X} \tau} \int_{0}^{\infty} \frac{\rho_{0} c / H_{0}}{\sqrt{\Omega_{m}(1+z)^{3}+\left(1-\Omega_{m}\right)}} \frac{d N_{\nu}}{d E_{\nu}}\left(E_{\nu}^{\prime}\right) d z
$$


where $c / H_{0}=1.37 \cdot 10^{28} \mathrm{~cm}$ is the Hubble length, $\rho_{0}=1.15 \cdot 10^{-6} \mathrm{GeV} / \mathrm{cm}^{3}$ is the average cosmological dark matter density for today, $\Omega_{m}=0.27$ and the injected spectrum $\frac{d N_{\nu}}{d E_{\nu}}$ is taken as a function of neutrino energy at redshift $z: E_{\nu}^{\prime}=E_{\nu}(1+z)$.

\section{Analysis \& discussion}

The method of constraining the dark-matter parameters with neutrino limits slightly differs from that using with the gamma-ray limits. The exposure of neutrino observatory depends on the neutrino energy, therefore flux limits depend on neutrino spectrum. Below we briefly describe the method. The quantity one needs to compare with the observation is the total number of neutrino events that would be detected in the given experiment under the assumption of the given neutrino spectrum. The method of calculation of this quantity was described in Ref. [51. Below all the quantities are related to neutrino therefore the index $\nu$ is omitted. For the galactic neutrino flux one has

$$
N_{\mathrm{G}}=\frac{1}{4 \pi M_{X} \tau} \int_{\Delta E} \int_{V} \rho[R(r, \delta, \alpha)] \varepsilon(E, \delta, \alpha) \frac{d N}{d E}(E) \cos (\delta) d r d \delta d \alpha d E ;
$$

where $\rho[R]$ is a dark matter density as a function of distance from the Galactic Center $R, r$ is a distance from Earth, $\varepsilon$ is the exposure of the given observatory as a function of the neutrino energy $E$ and equatorial coordinates $\{\delta, \alpha\}$. The integration takes over all volume of the dark-matter halo $(R<260 \mathrm{kpc})$ and over the entire range $\Delta E$ of the neutrino energies accessible for a given observatory. In practice, the exposure is given for several bands of zenith angle, averaged over each band. For IceCube we adopt the exposure as a function of declination (which uniquely translates to zenith angle in the case of IceCube) and energy as it is given in Ref. [52] and normalize it to the actual IceCube exposure of Ref. [39]. For Pierre Auger we use the exposure given in Ref. [40] together with the formula of the effective exposure of extensive air shower observatory [53, 54]:

$$
\omega\left(a_{0}, \delta, \theta_{\max }\right) \sim\left(\cos a_{0} \cos \delta \sin \alpha_{m}+\alpha_{m} \sin a_{0} \sin \delta\right),
$$

where $a_{0}$ is the geographical latitude of the given observatory, $\theta_{\max }$ is the maximal zenith angle accessible for fully efficient observation in this observatory and $\alpha_{m}$ is given by

$$
\begin{gathered}
\alpha_{m}= \begin{cases}0 & ; \xi>1, \\
\pi & ; \xi<-1, \\
\arccos \xi & ;-1<\xi<1\end{cases} \\
\xi=\frac{\left(\cos \theta_{\max }-\sin a_{0} \sin \delta\right)}{\cos a_{0} \cos \delta} .
\end{gathered}
$$

The number of events from the extragalactic flux is

$$
N_{\mathrm{EG}}=\int_{\Delta E} \varepsilon(E) \frac{d N_{\mathrm{EG}}}{d E}(E) d E
$$




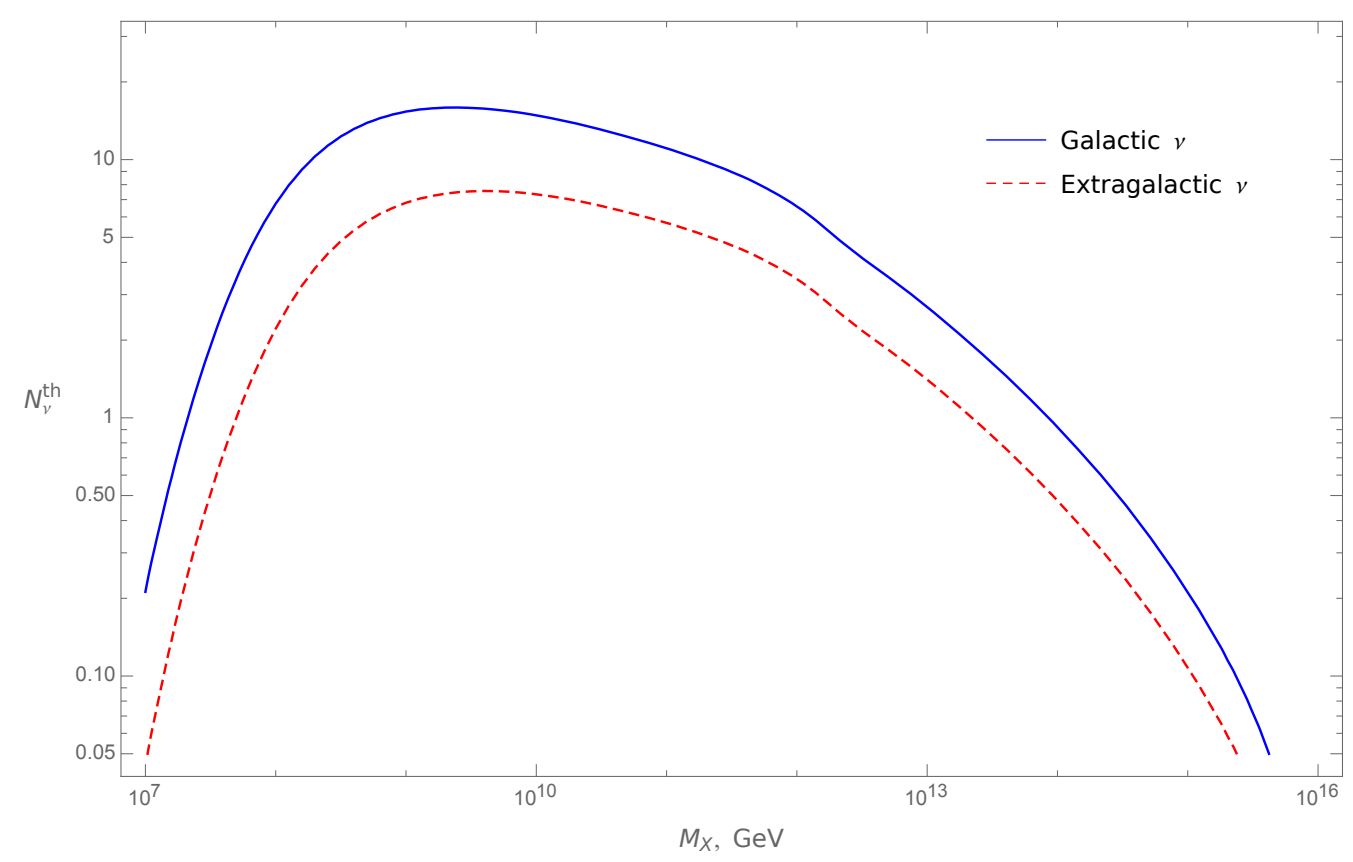

Figure 2: Total number of galactic (solid line) and extragalactic (dashed line) neutrinos from decays of dark matter particles with various masses $M_{X}$ and lifetime $\tau=10^{20} \mathrm{yr}$ as it could be received by the IceCube experiment.

where the exposure $\varepsilon(E)$ is integrated over the celestial sphere. Thus the total number of events predicted by the theory is

$$
N_{\mathrm{th}}=N_{\mathrm{G}}+N_{\mathrm{EG}} .
$$

The example of $N_{\mathrm{G}}$ and $N_{\mathrm{EG}}$ for fixed $\tau$ and various masses $M_{X}$ is shown in Fig. 2. There are two factors of resulting neutrino signal enhancement. One is due to the observation of the galactic flux, which exceeds the contribution of the rest of the Universe as one can learn from Fig. 2. Another one is due to the fact that largest high-energy neutrino observatories - IceCube and Pierre Auger can observe the enhanced neutrino flux from the Galactic Center region which is located in the southern sky.

For each mass $M_{X}$ the lifetime $\tau$ is subject to constrain. The standard technique of Ref. [55] implies that we vary $\tau$ until the predicted number of events $N_{\text {th }}$ reaches from below the number $N_{\text {limit }}$ specified for a given number of observed events $N_{\text {obs }}$, number of background events $N_{\text {bg }}$ and given confidence level. We may calculate $N_{\text {th }}$ over full range of accessible energies or in separate energy intervals. In the latter case the constraints on parameter $\tau$ can be weaker, since the number $N_{\text {limit }}$ does not depend on the length of the energy interval. In the case when $N_{\text {bg }}=0$ and $N_{\text {obs }} \neq 0$, the other method is more appropriate. We split the full energy range in separate intervals $\Delta E^{i}$ with certain $N_{\text {obs }}^{i}$ in each one and generate Monte-Carlo set which places in the $i$-th interval the number of events $N_{\mathrm{MC}}^{i}$ following the Poisson distribution with the mean $\lambda^{i}=N_{\mathrm{th}}^{i}$, the theoretical number of events calculated in the respective energy interval $\Delta E^{i}$. For each particular value of the parameter $\tau$ we generate a large number of these Monte-Carlo realisations. Then we vary the parameter $\tau$ until the fraction of realisations where $N_{\mathrm{MC}}^{i}>N_{\mathrm{obs}}^{i}$ at least in one bin reaches the given confidence level C.L. In the case of all $N_{\text {obs }}^{i}=$ 




Figure 3: All-sky averaged neutrino fluxes from decays of dark-matter particles with masses $M_{X}=10^{9}$ and $M_{X}=10^{12} \mathrm{GeV}$ and marginally allowed lifetime $\left(\tau=6.6 \cdot 10^{20}\right.$ and $\tau=3.5 \cdot 10^{20}$ yr respectively) compared with various models of astrophysical [59] (solid black) and cosmogenic [56 58] neutrino fluxes (the sum of neutrino and antineutrino of all flavours).

0 this method yields the same results as the Feldman-Cousins technique. While for $N_{\text {obs }}>0$ the constraints of the Monte-Carlo method appears somewhat stronger. In the IceCube dataset we neglect the background of $0.064_{-0.039}^{+0.023}$ atmospheric neutrino events and therefore can apply the described method.

The constraints on the parameter space $\left\{M_{X}, \tau\right\}$ are presented in Fig. 5 together with the constraints of works [24,38] as well as the gamma-ray constraints obtained in our previous work [41]. We should note that the present constraints are conservative since we consider the total predicted neutrino flux as a product of the dark-matter decay and do not allow for the possible astrophysical or cosmogenic contribution. One can see that the gamma-ray constraints overlap the neutrino ones in almost all dark-matter mass range except the narrow region around $M_{X} \sim 10^{8} \mathrm{GeV}$, where the neutrino constraints is slightly stronger. Nevertheless neutrino observation remains a crucial tool for the dark-matter indirect detection. For example, in the model of hadronically decaying dark matter considered in this paper and in our previous work [41] the ratio of neutrino flux to photon flux have the certain value $r$ which variates in the range $0.8 \lesssim r \lesssim 1.8$ depending on energy and $M_{X}$. This ratio could be an additional criterion for distinguishing between various hypotheses of photon and neutrino fluxes origin.

Some examples of neutrino fluxes from the decay of the dark matter with the marginally allowed lifetime are shown in Figs. 3 - 4 together with some competing astrophysical and cosmogenic neutrino fluxes. In Fig. 3 the all-sky averaged fluxes are given, while in Fig. 4 we show the fluxes coming from the several directions related to our Galaxy. One can see that it is hard to distinguish the all-sky averaged fluxes of dark-matter decay from 


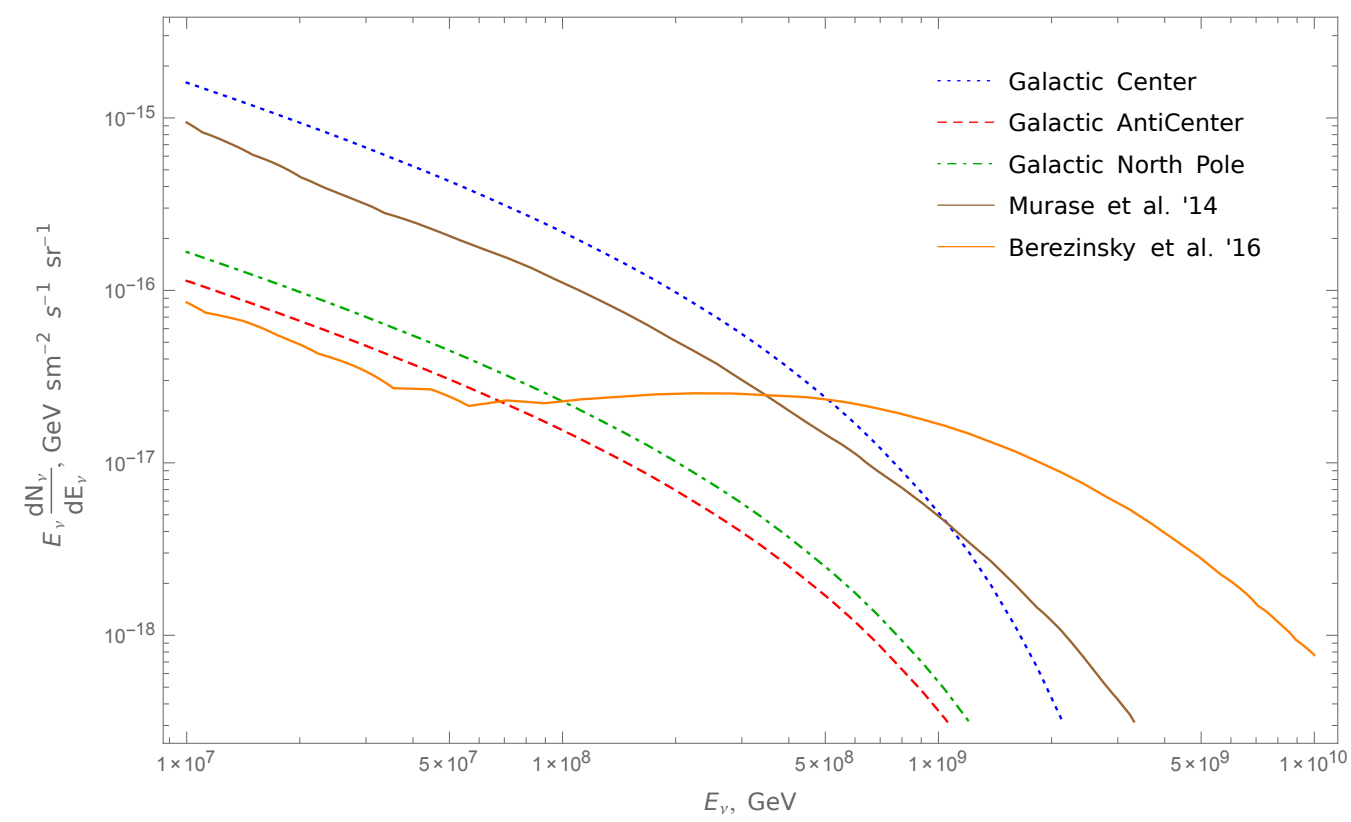

Figure 4: Comparison of neutrino fluxes from decays of dark-matter particles with mass $M_{X}=10^{10} \mathrm{GeV}$ and marginally allowed lifetime $\tau=7.75 \cdot 10^{20} \mathrm{yr}$, coming from several directions with one model of astrophysical neutrino flux [59] (solid brown) and one model of cosmogenic one [56] (solid orange) (the sum of neutrino and antineutrino of all flavours).

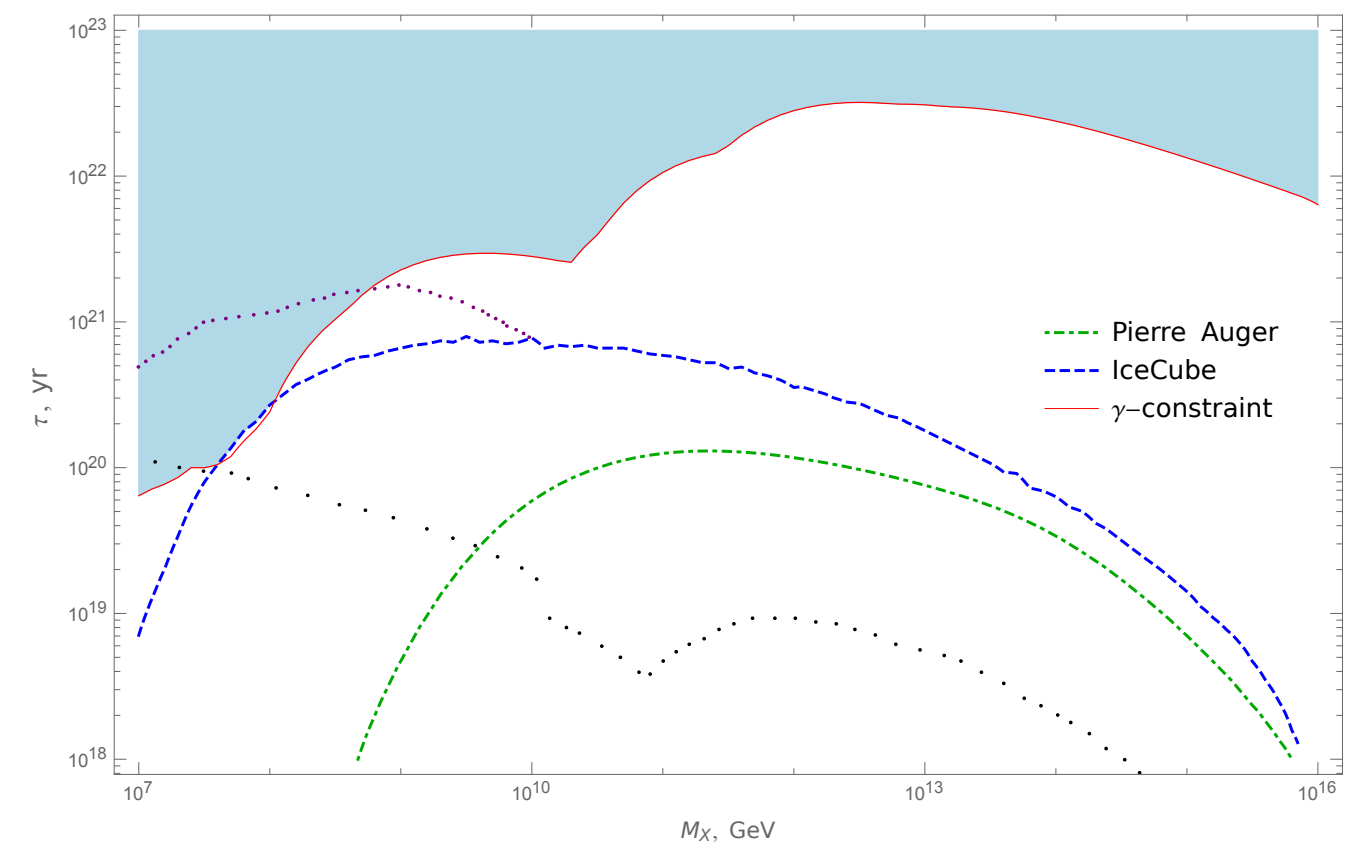

Figure 5: $90 \%$ C.L. exclusion plot for mass $M_{X}$ and lifetime $\tau$ of dark-matter particles. White area is excluded. For comparison we present the constraints obtained with photon limits [41] (solid thin red line). We also show the constraint obtained in the dark matter model with $X \rightarrow \nu \bar{\nu}$ decay channel [38] (black dots) and constraint for $X \rightarrow b \bar{b}$ channel which assumes that the IceCube events are of astrophysical origin [24] (purple dots). 
astrophysical and cosmogenic ones. However, this problem simplifies when we compare the directional fluxes ${ }^{1}$ ). Therefore the analysis of the Galactic anisotropy of the signal become crucial for the dark matter indirect search.

\section{Conclusion}

The implications of the new IceCube dataset of high energy neutrino to the hadronically decaying heavy dark matter theory was considered. It was found that for the dark matter masses $10^{7} \leq M_{X} \leq 10^{16} \mathrm{GeV}$ the neutrino data bound dark matter lifetime stronger than the gamma-ray limits of the extensive air shower observatories only in the narrow region around $M_{X} \sim 10^{8} \mathrm{GeV}$. One of the reasons of this fact is that photon exposures of experiments are typically larger than its neutrino exposures. It is also meaningful that the non-zero flux of high energy neutrino was observed, contrary to the non-observation of photons of the same energies. It was emphasized that the relevant test for distinguishing the signal of the decaying dark matter from signals of other origin is the analysis of the galactic anisotropy and photon-neutrino flux ratio.

\section{Acknowledgements}

I would like to thank S. Troitsky, G. Rubtsov, O. Kalashev and D. Gorbunov for helpful discussions. I am especially indebted to R. Aloisio, V. Berezinsky and M. Kachelriess for providing the numerical code solving the DGLAP equations. This work has been supported by the Russian Science Foundation grant 14-12-01340.

\section{References}

[1] Ya. B. Zeldovich and A. A. Starobinsky, Sov. Phys. JETP 34, 1159 (1972) [Zh. Eksp. Teor. Fiz. 61, 2161 (1971)].

[2] Ya. B. Zeldovich and A. A. Starobinsky, JETP Lett. 26, 252 (1977) [Pisma Zh. Eksp. Teor. Fiz. 26, 373 (1977)].

[3] V. A. Kuzmin and V. A. Rubakov, Phys. Atom. Nucl. 61, 1028 (1998) astroph/9709187.

[4] V. Berezinsky, M. Kachelriess and A. Vilenkin, Phys. Rev. Lett. 79, 4302 (1997) astro-ph/9708217].

[5] L. Kofman, A. D. Linde and A. A. Starobinsky, Phys. Rev. Lett. 73, 3195 (1994) hep-th/9405187.

\footnotetext{
1) There is a subtlety related to the choice of dark matter profile. Navarro-Frenk-White profile produces the larger signal from the Galactic Center direction compared to the cored profiles. For example, the GC flux in Burkert profile [60] is approximately 3 times smaller than that of NFW, while the difference between the overall fluxes is negligible.
} 
[6] S. Y. Khlebnikov and I. I. Tkachev, Phys. Rev. Lett. 79, 1607 (1997) hepph/9610477.

[7] S. Y. Khlebnikov and I. I. Tkachev, Phys. Lett. B 390, 80 (1997) hep-ph/9608458.

[8] V. Kuzmin and I. Tkachev, Phys. Rev. D 59, 123006 (1999) [hep-ph/9809547].

[9] D. J. H. Chung, E. W. Kolb and A. Riotto, Phys. Rev. D 59, 023501 (1999) hepph/9802238.

[10] D. J. H. Chung, E. W. Kolb and A. Riotto, Phys. Rev. D 60, 063504 (1999) hepph/9809453.

[11] V. Kuzmin and I. Tkachev, JETP Lett. 68, 271 (1998) [Pisma Zh. Eksp. Teor. Fiz. 68, 255 (1998)] [hep-ph/9802304].

[12] D. Fargion, M. Y. Khlopov, R. V. Konoplich, V. R. Konoplich and R. Mignani, Mod. Phys. Lett. A 11, 1363 (1996).

[13] M. Y. Khlopov and V. M. Chechetkin, Fiz. Elem. Chast. Atom. Yadra 18, 627 (1987).

[14] P. Gondolo, G. Gelmini and S. Sarkar, Nucl. Phys. B 392, 111 (1993) hepph/9209236.

[15] T. Abu-Zayyad et al. [Telescope Array Collaboration], Astrophys. J. 768, L1 (2013) arXiv:1205.5067 [astro-ph.HE]].

[16] J. Abraham et al. [Pierre Auger Collaboration], Phys. Rev. Lett. 101, 061101 (2008) arXiv:0806.4302 [astro-ph]].

[17] D. S. Gorbunov and V. A. Rubakov, "Introduction to the theory of the early universe: Hot big bang theory," Hackensack, NJ : World Sci., 2011

[18] E. W. Kolb, D. J. H. Chung and A. Riotto, In *Heidelberg 1998, Dark matter in astrophysics and particle physics 1998* 592-614 hep-ph/9810361.

[19] V. A. Kuzmin and I. I. Tkachev, Phys. Rept. 320, 199 (1999) hep-ph/9903542].

[20] D. J. H. Chung, E. W. Kolb, A. Riotto and L. Senatore, Phys. Rev. D 72 (2005) 023511 astro-ph/0411468.

[21] D. S. Gorbunov and A. G. Panin, Phys. Lett. B 718, 15 (2012) arXiv:1201.3539 [astro-ph.CO]].

[22] O. E. Kalashev, G. I. Rubtsov and S. V. Troitsky, Phys. Rev. D 80, 103006 (2009) arXiv:0812.1020 [astro-ph]].

[23] K. Murase and J. F. Beacom, JCAP 1210, 043 (2012) arXiv:1206.2595 [hep-ph]].

[24] T. Cohen, K. Murase, N. L. Rodd, B. R. Safdi and Y. Soreq, arXiv:1612.05638 [hep-ph]. 
[25] R. Aloisio, S. Matarrese and A. V. Olinto, JCAP 1508, no. 08, 024 (2015) arXiv:1504.01319 [astro-ph.HE]].

[26] A. Esmaili and P. D. Serpico, JCAP 1510, no. 10, 014 (2015) arXiv:1505.06486 [hep-ph]].

[27] M. G. Aartsen et al. [IceCube Collaboration], Science 342, 1242856 (2013) arXiv:1311.5238 [astro-ph.HE]].

[28] M. G. Aartsen et al. [IceCube Collaboration], Phys. Rev. Lett. 113, 101101 (2014) arXiv:1405.5303 [astro-ph.HE]].

[29] O. E. Kalashev and S. V. Troitsky, JETP Lett. 100, no. 12, 761 (2015) [Pisma Zh. Eksp. Teor. Fiz. 100, no. 12, 865 (2014)] arXiv:1410.2600 [astro-ph.HE]].

[30] M. G. Aartsen et al. [IceCube Collaboration], Phys. Rev. Lett. 115, no. 8, 081102 (2015) arXiv:1507.04005 [astro-ph.HE]].

[31] C. D. Dermer, K. Murase and Y. Inoue, JHEAp 3-4, 29 (2014) arXiv:1406.2633 [astro-ph.HE]].

[32] A. Bhattacharya, M. H. Reno and I. Sarcevic, JHEP 1406, 110 (2014) arXiv:1403.1862 [hep-ph]].

[33] P. S. B. Dev, D. Kazanas, R. N. Mohapatra, V. L. Teplitz and Y. Zhang, JCAP 1608, no. 08, 034 (2016) arXiv:1606.04517 [hep-ph]].

[34] A. Esmaili and P. D. Serpico, JCAP 1311, 054 (2013) [arXiv:1308.1105 [hep-ph]].

[35] A. Esmaili, S. K. Kang and P. D. Serpico, JCAP 1412, no. 12, 054 (2014) arXiv:1410.5979 [hep-ph]].

[36] K. Murase, R. Laha, S. Ando and M. Ahlers, Phys. Rev. Lett. 115, no. 7, 071301 (2015) arXiv:1503.04663 [hep-ph]].

[37] C. Rott, K. Kohri and S. C. Park, Phys. Rev. D 92, no. 2, 023529 (2015) arXiv:1408.4575 [hep-ph]].

[38] A. Esmaili, A. Ibarra and O. L. G. Peres, JCAP 1211, 034 (2012) arXiv:1205.5281 [hep-ph]].

[39] M. G. Aartsen et al. [IceCube Collaboration], Phys. Rev. Lett. 117, no. 24, 241101 (2016) arXiv:1607.05886 [astro-ph.HE]].

[40] A. Aab et al. [Pierre Auger Collaboration], Phys. Rev. D 91, no. 9, 092008 (2015) arXiv:1504.05397 [astro-ph.HE]].

[41] O. E. Kalashev and M. Y. Kuznetsov, Phys. Rev. D 94, no. 6, 063535 (2016) arXiv:1606.07354 [astro-ph.HE]].

[42] M. Cirelli et al., JCAP 1103, 051 (2011) Erratum: [JCAP 1210, E01 (2012)] arXiv:1012.4515 [hep-ph]]. 
[43] R. Aloisio, V. Berezinsky and M. Kachelriess, Phys. Rev. D 69, 094023 (2004) hepph/0307279|.

[44] S. Sarkar and R. Toldra, Nucl. Phys. B 621, 495 (2002) hep-ph/0108098.

[45] M. Hirai, S. Kumano, T.-H. Nagai and K. Sudoh, Phys. Rev. D 75, 094009 (2007) hep-ph/0702250].

[46] V.N. Gribov and L.N. Lipatov, Sov. J. Nucl. Phys. 15, 438 (1972); L.N. Lipatov, Sov. J. Nucl. Phys. 20, 94 (1975); Yu. L. Dokshitzer, Sov. Phys. JETP 46, 641 (1977).

[47] G. Altarelli and G. Parisi, Nucl. Phys. B126, 298 (1977).

[48] S. R. Kelner, F. A. Aharonian and V. V. Bugayov, Phys. Rev. D 74, 034018 (2006) Erratum: [Phys. Rev. D 79, 039901 (2009)] [astro-ph/0606058].

[49] J. F. Navarro, C. S. Frenk and S. D. M. White, Astrophys. J. 462, 563 (1996) astro-ph/9508025].

[50] J. F. Navarro, C. S. Frenk and S. D. M. White, Astrophys. J. 490, 493 (1997) astro-ph/9611107.

[51] L. A. Anchordoqui, J. L. Feng, H. Goldberg and A. D. Shapere, Phys. Rev. D 66, 103002 (2002) [hep-ph/0207139].

[52] R. Abbasi et al. [IceCube Collaboration], Phys. Rev. D 83, 092003 (2011) Erratum: [Phys. Rev. D 84, 079902 (2011)] [arXiv:1103.4250 [astro-ph.CO]].

[53] P. Sommers, Astropart. Phys. 14, 271 (2001) astro-ph/0004016.

[54] A. Aab et al. [Telescope Array and Pierre Auger Collaborations], Astrophys. J. 794, no. 2, 172 (2014) arXiv:1409.3128 [astro-ph.HE]].

[55] G. J. Feldman and R. D. Cousins, Phys. Rev. D 57, 3873 (1998) physics/9711021 [physics.data-an]].

[56] V. Berezinsky, A. Gazizov and O. Kalashev, Astropart. Phys. 84, 52 (2016) arXiv:1606.09293 [astro-ph.HE]].

[57] M. Ahlers, L. A. Anchordoqui, M. C. Gonzalez-Garcia, F. Halzen and S. Sarkar, Astropart. Phys. 34, 106 (2010) [arXiv:1005.2620 [astro-ph.HE]].

[58] K. Kotera, D. Allard and A. V. Olinto, JCAP 1010, 013 (2010) arXiv:1009.1382 [astro-ph.HE]].

[59] K. Murase, Y. Inoue and C. D. Dermer, Phys. Rev. D 90, no. 2, 023007 (2014) arXiv:1403.4089 [astro-ph.HE]].

[60] A. Burkert, IAU Symp. 171, 175 (1996) [Astrophys. J. 447, L25 (1995)] astroph/9504041. 